\title{
Research on the Meteorological Prediction Algorithm Based on the CNSS and Particle Swarm Optimization
}

\author{
Li Yang, Meng Zhang $(D$, and Yunhan Zhang \\ College of Environment and Planning, Henan University, Kaifeng 475000, Henan, China \\ Correspondence should be addressed to Meng Zhang; 104754190142@henu.edu.cn
}

Received 14 May 2021; Accepted 24 June 2021; Published 2 July 2021

Academic Editor: Huihua Chen

Copyright ( $2021 \mathrm{Li}$ Yang et al. This is an open access article distributed under the Creative Commons Attribution License, which permits unrestricted use, distribution, and reproduction in any medium, provided the original work is properly cited.

\begin{abstract}
Considering that the global navigation satellite system (GNSS) has the influence of positioning and atmospheric signals from time to time in meteorology, errors caused by moisture, and so on in the effect of the propagation path, these factors have led to the influence of various indexes of meteorological factors. In this study, a meteorological prediction algorithm based on the CNSS and particle swarm optimization is proposed. Aiming at the phenomenon that the particle swarm optimization (PSO) algorithm is prone to slow convergence speed and low optimization accuracy and there is a local optimal but cannot achieve the global optimal, an adaptive Kent chaotic map PSO algorithm is proposed. Through the comprehensive analysis of the meteorological input indicators in the GNSS, a noncurrent weight evaluation system is proposed. Under different evaluation systems, the PSO algorithm is applied, and PCA weight can obtain the best prediction effect. Then, the GA model, PSO model, and ADPSO model are used to predict PM2.5 index in meteorology. The results show that the proposed ADPSO algorithm has a good performance in RMSE, MAE, and $R^{2}$ model evaluation.
\end{abstract}

\section{Introduction}

The GNSS has the characteristics of omnibearing, allweather, all-time, and high precision. During its positioning, when GNSS signals pass through Earth's atmosphere, they will be affected by the combined action of ionosphere and atmosphere, which will slow down the signal propagation speed and bend the propagation path, causing a delay in time. This delay in time is equivalent to the growth of the propagation path. It becomes an error source in GNSS positioning [1], in which ionospheric errors can be eliminated by receiving dual-frequency signals and linearly combining them. The total tropospheric delay in the zenith direction is approximately 2.5 meters. The main reason is dry delay caused by dry air, about $90 \%$. The proportion of wet delay caused by water vapor is small, about $10 \%$; water vapor only accounts for $0.1-4 \%$ of the whole atmospheric composition. It is an important part of the atmosphere, which is used to describe the atmospheric state. Although the content of water vapor in the air is low, the wet delay caused by water vapor changes rapidly, which makes it difficult to estimate the wet delay effectively. It is a key error that cannot be ignored in the GNSS positioning error source. Researchers can retrieve the water vapor in the atmosphere by using this error source [2].

Conventional detection methods of atmospheric water vapor include radiosonde technology, satellite remote sensing inversion, microwave radiometer measurement, and radar detection. Radiosonde and microwave radiometer are complicated and expensive to measure. However, the accuracy of satellite remote sensing inversion is low and cannot meet the needs of daily and scientific research. Therefore, new atmospheric water vapor detection technology is urgently needed to obtain accurate atmospheric data [3]. Duan et al. proposed a method of directly measuring GPS absolute precipitable water in 1996 [4]. Since then, Boehm et al. have performed a lot of work to promote the development of GPS meteorology [5, 6]. Literature [7] proposes that the GNSS prediction value is used to predict weather forecast, and STD is assimilated into numerical forecast by using temporal and spatial correlation in the GNSS. Composite observations obtained from numerical 
weather prediction model fields are used to evaluate the influence of additional artificial factors (ionosphere, clock, ambiguity, and multipath) on tropospheric estimates. Literature [8] uses the comparative advantage of GNSS observations in large time to quickly obtain clear humidity information from ZTD. The forecast is carried out for specific areas, with emphasis on evaluating the correlation between humidity and wind. The GNSS, as an uninterrupted provision of corresponding timely observation values, has great advantages in prediction accuracy. Literature [9] studies and discusses the influence of ZTD weather forecast analysis obtained by the GNSS network and subsequent forecast quality. ZTD has obvious positive effects on water vapor in the middle and lower troposphere, air temperature in the upper troposphere, and wind in the middle and upper troposphere. Compared with the model without ZTD assimilation, the error is reduced by $4 \%$. The advantages of ZTD in the GNSS for improving weather forecast are evaluated, and the construction of the GNSS network is enhanced. Document [10] proposes an INCA system for measuring the GNSS tropospheric water vapor content for areas where climate conditions change rapidly and are difficult to predict or areas with rugged terrain and great height differences, with a time resolution of $1 \mathrm{~h}$ and a time delay of less than $1 \mathrm{~h}$. The results show that the accuracy of the estimated value of precipitable water $(\mathrm{PW})$ is better than $1 \mathrm{~mm}$. In [11], the GNSS-RO system monitors climate, atmosphere, ionosphere, and earth. Relevant experiments show that CNSS-RO can improve the accuracy of hurricane/ typhoon behavior prediction and significantly improve longterm weather prediction. Document [12] evaluates the application effect of RNSSs and GNSSs in space monitoring, citing GPS radio occultation (RO) data to better simulate climate models, forecast weather, and monitor ionosphere. Document [13] proposes a GNSS/PWV dynamic meteorological monitoring system based on Matlab platform. Considering PWV, temperature, and humidity in meteorological factors, a time series analysis model and a plane dynamic visualization model are established. The customer can reflect that the system can predict the one-dimensional and two-dimensional water vapor dynamic changes of the weather and improve the weather prediction ability and monitoring level. Literature [14] studies the causes of evaluation errors in view of the uncertainty of GNSS prediction and evaluation of water vapor in the atmosphere. Statistical analysis and theoretical analysis are used to evaluate the uncertain factors of water vapor. The input variables, software and hardware, data processing, and data point allocation are emphatically evaluated, and the maximum uncertain factor is determined to be $75 \%$ in ZTD. The conversion factors of water vapor and ZTD are positively correlated, and the proportion increases in humid weather. Literature [15] puts forward a method of using GNSS observation data to predict precipitation in meteorology and uses the current GNSS precipitation to predict this result. Differential GNSS measurement is used to reduce GNSS and clock errors, and meteorological measurement data and delay accuracy GNSS PWV are used to evaluate system performance. The results show that the error of real-time
PWV is $2.1 \sim 3.4 \mathrm{~mm}$, and the difference error is $1.4 \sim 2.9 \mathrm{~mm}$ after reducing the comparison period for 20 days, which has a high prediction effect. Document [16] effectively fuses the input GNSS and InSAR data, geodetic data, and meteorological data. The neutral layer phase of InSAR is separated from the components in InSAR measurement. In the comparative study of the GNSS, there are related observation residuals such as the prediction model, mapping function, and gradient parameters in the GNSS. In addition, the influence of InSAR residual on information path is planned, and the midstream layer derived from weather model data is analyzed suburban. Literature [17] selects correlation prediction values from GNSS stations and shows that there is $1 \%$ correlation between GNSS and PWV from linear relationship. Combining PWV in the GNSS with meteorological data, Bayesian and Levenberg Marquardt algorithms are used to predict $30 \%$ and $50 \%$ of the dataset. It is beneficial to the real-time monitoring of meteorology by the system and can improve the prediction accuracy. The particle swarm optimization algorithm based on improved self-application proposed in this study is applied to predict meteorological information. In this study, meteorological information is predicted according to the concentration values of $\mathrm{PM}_{2.5}, \mathrm{PM}_{10}$, and $\mathrm{CO}$. Tables 1 and 2 have various input indexes. The performance of the algorithm is evaluated as an index, the optimal effect is obtained, and the performance is compared with other algorithms again.

The second part of this study introduces the GNSS meteorological information model and PSO model. The third part describes the implementation process of the selfapplied PSO algorithm. The fourth part evaluates the performance of the algorithm by using four weight relationships. In the fifth part, under the optimal weight evaluation, different algorithms are compared. The effective prediction of weather can solve the problem of the poor prediction effect and can timely understand the space quality and corresponding climate problems.

\section{Introduction of Relevant Theories}

2.1. GNSS Meteorological Correlation Model. GNSS stream layer delay can be described by the data model [18], and relevant indexes can be obtained by studying delay data. The tropospheric delay is described by the length of the propagation path, and the formula is as follows:

$$
\Delta L=\int_{L} n(s) \mathrm{d} s-G .
$$

There is a refractive index in the atmosphere on the path $L, G$ is the physical length between the satellite and the receiver, and equation (1) can be changed as follows:

$$
\Delta L=\int_{L}[n(s)-1] \mathrm{d} s-(S-G) .
$$

In equation (2), $S-G$ represents the influence of signal path bending. The value is relatively small, and the delay is generally negligible. Considering the relatively small value $(n-1), N$ represents the atmospheric refractive index, which 
TABLE 1: Input of meteorological factors.

\begin{tabular}{lcc}
\hline Variable & Symbol & Unit \\
\hline Daily precipitation & $R$ & $\mathrm{~mm}$ \\
Sunshine time & $S$ & $\mathrm{~h}$ \\
Daily maximum temperature & $T_{\max }$ & $\mathrm{C}$ \\
Daily minimum temperature & $T_{\min }$ & $\mathrm{C}$ \\
Average air temperature & $T_{\text {ave }}$ & $\mathrm{C}$ \\
Minimum relative humidity & $H_{\min }$ & $\%$ \\
Average relative humidity & $H_{\text {ave }}$ & $\%$ \\
Large evaporation & $E_{l}$ & $\mathrm{~mm}$ \\
Small evaporation & $E_{s}$ & $\mathrm{~mm}$ \\
Maximum wind speed & $V_{\max }$ & $\mathrm{m} / \mathrm{s}$ \\
Wind direction of maximum wind speed & $\mathrm{WD}_{\max }$ & $\mathrm{Azimuth}$ \\
Maximum wind speed & $V_{10-\mathrm{ave}}$ & $\mathrm{m} / \mathrm{s}$ \\
Wind direction of maximum wind speed & $\mathrm{WD}_{10-\mathrm{ave}}$ & $\mathrm{Azimuth}$ \\
Average wind speed & $V_{\mathrm{ave}}$ & $\mathrm{m} / \mathrm{s}$ \\
Mean water vapor pressure & $V_{P}$ & $\mathrm{hPa}$ \\
Daily maximum pressure & $P_{\max }$ & $\mathrm{hPa}$ \\
Daily minimum pressure & $P_{\min }$ & $\mathrm{hPa}$ \\
Daily mean pressure & $P_{\text {ave }}$ & $\mathrm{hPa}$ \\
\hline
\end{tabular}

TABLE 2: Input of basic value of pollutant mass concentration.

\begin{tabular}{lcc}
\hline Variable name & Variable symbol & $\mathrm{Unit}$ \\
\hline Mass concentration of $\mathrm{PM}_{2.5}$ & $V_{\mathrm{pm} 2.5}$ & $\mathrm{G} / \mathrm{m}^{3}$ \\
Mass concentration of $\mathrm{PM}_{10}$ & $V_{\mathrm{pm} 10}$ & $\mathrm{G} / \mathrm{m}^{3}$ \\
CO mass concentration & $V_{\mathrm{co}}$ & $\mathrm{G} / \mathrm{m}^{3}$ \\
$\mathrm{O}_{3}$ mass concentration & $V_{o 3}$ & $\mathrm{G} / \mathrm{m}^{3}$ \\
$\mathrm{NO}_{2}$ mass concentration & $V_{n o 3}$ & $\mathrm{G} / \mathrm{m}^{3}$ \\
$\mathrm{SO}_{2}$ mass concentration & $V_{s o 2}$ & $\mathrm{G} / \mathrm{m}^{3}$ \\
\hline
\end{tabular}

is $106(n-1)$. The related formula of total tropospheric delay can be described as follows:

$$
\Delta L=\int_{L}[n(s)-1] \mathrm{d} s-(S-G)=\Delta L_{s h}+\Delta L_{s w}+\Delta L_{s m},
$$

where $\Delta L_{s h}$ and $\Delta L_{s w}$ denote the static and wet retardation, respectively. $\Delta L_{s m}$ indicates the delay of water vapor condensate in oblique path.

The total delay of the oblique path can be projected to the zenith direction of the station through the mapping function to obtain the average value of the total delay on multiple rays in the zenith direction, as shown in the following formula:

$$
D_{\mathrm{zt}}=D_{\mathrm{st}} \cdot f\left(e_{\mathrm{el}}, i_{\mathrm{az}}\right),
$$

where $D_{\mathrm{zt}}$ represents the total zenith delay, $e_{\mathrm{al}}$ and $i_{\mathrm{az}}$ represent the altitude angle and azimuth angle of the satellite, respectively, and $f$ is the projection function. On the basis of ensuring a certain accuracy, the satellite signal rays with different altitude angles are converted to the zenith direction, and the influence of satellite signal bending is ignored. When projected to the zenith, the expression is

$$
D_{\mathrm{zt}}=D_{\mathrm{zh}}+D_{\mathrm{zw}}+D_{\mathrm{zhw}} \text {. }
$$

where $D_{\mathrm{zw}}$ is the zenith wet delay, and the main change is affected by the water vapor content over the station. $D_{z h m}$ is the zenith water vapor condensation delay.
2.2. Basic Particle Swarm Optimization (PSO). The PSO algorithm is based on the simulation process of the predation behavior of animals and birds. In the whole space, each bird moves, can capture the optimal position of food in the space and the global optimal position of food that all birds can hunt. Birds can adjust their flight speed and position according to their position in the global situation [19] to obtain the behavior of the overall optimal food position.

Let the initial bird population be $N$ in $\mathrm{D}$-dimensional space, the position of the $i^{\text {th }}$ particle is $x_{i}=$ $\left[x_{i 1}, x_{i 2}, \ldots, x_{i D}\right]$, and the flight speed is $v_{i}=$ $\left[v_{i 1}, v_{i 2}, \ldots, v_{i D}\right]$. When the population undergoes $t$ optimization iterations, the optimal position of the particles is obtained $p_{\text {best }}$, and the global optimal position of the particle is $g_{\text {best }}$. Then, the formula for calculating the particle flight speed and position update is expressed as follows:

$$
v_{i, d}^{t+1}=w v_{i, d}^{t}+c_{1} r_{1}\left(\text { pbest }_{i, d}^{t}-x_{i, d}^{t}\right)+c_{2} r_{2}\left(\text { gbest }_{d}^{t}-x_{i, d}^{t}\right),
$$

$$
x_{i, d}^{t+1}=x_{i, d}^{t}+v_{i, d}^{t+1},
$$

$$
\begin{aligned}
& \text { pbest }=\left(p_{i 1}, p_{i 2}, \ldots, p_{i D}\right), \\
& \text { gbest }=\left(p_{g 1}, p_{g 2}, \ldots, p_{g D}\right) .
\end{aligned}
$$

In the above equations (6)-(9), in the $i^{\text {th }}$ particle optimization process, $v_{i, d}^{t+1}$ refers to the velocity in D-dimensional space after $t+1$ iterations, $x_{i, d}^{t}$ refers to the position in $\mathrm{D}$-dimensional space after $t$ iterations, $w$ is an inertia weight, $c_{1}$ and $c_{2}$ are the learning factors, set $c_{1}=c_{2}=2$, and $r_{1}$ and $r_{2}$ are the random numbers between 0 and 1 .

Equation (6) updates the velocity of the latest particle at present, and equation (7) predicts that the new position information is based on the superposition of the last position and the current velocity. Equations (8) and (9) take local optimal particles and global optimal particles.

Formula (1) contains three formulas, which represent the corresponding special meanings in particle swarm optimization. The first formula $v_{i, d}^{t}$ indicates that particles search in the region according to the speed and direction of previous normal habits. The second module $c_{1} r_{1}\left(p_{\text {best }}^{t}{ }_{i, d}-x_{i, d}^{t}\right)$ indicates that particles work with the best search effect in history in the current space. The third module $c_{2} r_{2}$ (gbest ${ }_{d}^{t}-$ $\left.x_{i, d}^{t}\right)$ is that each particle has the habit of searching in the solution space following the optimal way of population history and imparting its historical experience to other people who want to acquire knowledge. Generally speaking, the particle swarm optimization algorithm is to find optimization by learning and imitating the way and historical behavior of creatures discovering things in nature. follows:

The basic particle swarm optimization algorithm is as

Step 1: initialize the population

Step 2: calculate the fitness of particles 
Step 3: update the global extreme value and the individual extreme value according to the fitness of the particles

Step 4: update the velocity and position of particles according to equations (6) and (9)

Step 5: determine whether the maximum iteration number is reached, if the program ends, and if it does not, enter Step 2.

\section{Adaptive Optimization Particle Swarm Optimization (ADPSO)}

Particle swarm optimization (PSO) has the advantages of the simple model, few adjusted parameters, and easy implementation, but it is easy to have slow convergence speed and low optimization accuracy in the optimization process of some complex functions. In view of the shortcomings of the PSO algorithm, the standard algorithm is improved.

\subsection{Initialization Population of Quantum Bloch Coordinate} Coding. In quantum computation, the smallest information unit is expressed by qubits. The state of a qubit can be expressed as [12]

$$
|\varphi\rangle=\cos (\theta / 2)|0\rangle+e^{i \varphi} \sin (\theta / 2)|1\rangle .
$$

In equation ( 3 ), the numbers $\theta$ and $\varphi$ sum defines a point $P$ on the Bloch sphere, as shown in Figure 1.

As is known from Figure 1, there is a one-to-one correspondence between quantum position and Bloch spherical point. Bloch coordinates are used to describe the quantum position:

$$
|\varphi\rangle=[\cos \varphi \sin \theta \sin \varphi \sin \theta \cos \theta]^{T} .
$$

Points on Bloch coordinates are used to describe the coding relationship of qubits, so that the $i^{\text {th }}$ candidate solution of $p_{i}$ population is described as follows:

$$
p_{i}=\left|\begin{array}{c|c|c}
\cos \varphi_{i 1} \sin \theta_{i 1} & \cdots & \cos \varphi_{i n} \sin \theta_{i d} \\
\sin \varphi_{i 1} \sin \theta_{i 1} & \ldots & \sin \varphi_{i n} \sin \theta_{i d} \\
\cos \theta_{i 1} & \ldots & \cos \theta_{i d}
\end{array}\right|,
$$

where $\varphi_{i j}=2 \pi \times \mathrm{rnd}, \theta_{i j}=\pi \times \mathrm{rnd}$, and rnd is a random number between 0 and $1 ; i=1,2, \ldots, n ; j=1,2, \ldots, d ; n$ is the size of the population, and $d$ is the dimension of the space. The three optimal solutions for candidate solutions in space are described as follows:

$$
\begin{aligned}
P_{i x} & =\left(\cos \varphi_{i 1} \sin \theta_{i 1}, \ldots, \cos \varphi_{i d} \sin \theta_{i d}\right), \\
P_{i y} & =\left(\sin \varphi_{i 1} \sin \theta_{i 1}, \ldots, \sin \varphi_{i d} \sin \theta_{i d}\right), \\
P_{i z} & =\left(\cos \theta_{i 1}, \cos \theta_{i 2}, \ldots, \cos \theta_{i d}\right) .
\end{aligned}
$$

The corresponding feasible solutions are

$$
\begin{aligned}
& x_{i x}=\left(x_{i 1}^{1}, x_{i 2}^{1}, \ldots, x_{i n}^{1}\right), \\
& x_{i y}=\left(x_{i 1}^{2}, x_{i 2}^{2}, \ldots, x_{i n}^{2}\right), \\
& x_{i z}=\left(x_{i 1}^{3}, x_{i 2}^{3}, \ldots, x_{i n}^{3}\right) .
\end{aligned}
$$

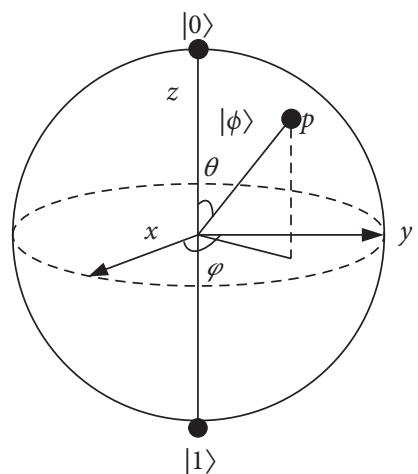

FIgURE 1: Bloch sphere representation of a qubit.

Solution space transformation: for Bloch coordinates $\left[x_{i x}, x_{i y}, x_{i z}\right]^{T}$ on $P_{i}$, find the corresponding qubits. In the optimization problem, the value range of the $j^{\text {th }}$ dimension of each solution space is $\left[a_{j}, b_{j}\right]$, and the solution space formula of unit space $I^{d}=[-1,1]^{d}$ is

$$
\begin{aligned}
& x_{i j}^{1}=\frac{1}{2}\left[b_{j}\left(1+x_{i x}\right)+a_{j}\left(1-x_{i x}\right)\right], \\
& x_{i j}^{2}=\frac{1}{2}\left[b_{j}\left(1+x_{i y}\right)+a_{j}\left(1-x_{i y}\right)\right], \\
& x_{i j}^{3}=\frac{1}{2}\left[b_{j}\left(1+x_{i z}\right)+a_{j}\left(1-x_{i z}\right)\right] .
\end{aligned}
$$

3.2. Optimization Strategy. The Kent chaotic map model is described as follows:

$$
Z^{t+1}= \begin{cases}\frac{Z^{t}}{a}, & 0<Z^{t} \leq a, \\ \frac{\left(1-Z^{t}\right)}{(1-a)}, & a<Z^{t}<1 .\end{cases}
$$

In formula (16), parameter $a \in(0,1)$; when Lyapunov $>0$ of Kent mapping, the mapping relationship is in a chaotic state. Set $a=0.4$, and the probability density function needs to obey uniform distribution, and its value is between 0 and 1. Lyapunov describes the divergence ratio of small uncertainties in the initial state. The Lyapunov is 0.696 for Kent chaos and 0.691 for logistic, which is dominant.

When chaos searches, the ergodicity of chaotic motion is used to generate chaotic series based on the current search stagnant solution, and the global optimization is obtained from the optimal solution of the whole sequence, so that the algorithm falls into local optimization. In PSO particle optimization, the optimal sequence of the solution space is not significantly improved after continuous limit iteration search, which shows that the global optimal solution is not easy to obtain in the local optimal solution of the solution space, so Kent is used for chaotic optimization. Chaotic optimization is carried out on the local optimal position of the PSO algorithm. The solution space of the optimization 
problem is $\left[X_{\min }, X_{\max }\right]$. Kent implementation steps are as follows:

Step 1 : the sums $X_{i j}^{1}, X_{i j}^{2}$, and $X_{i j}^{3}$ are mapped into the domain $[0,1]$ of Kent equation by using equation (16):

$$
Z^{0}=\frac{X_{\alpha}-X_{\min }}{X_{\max }-X_{\min }}
$$

Step 2 : generating $C_{\max }$ chaotic variable sequences by iteration of Kent equation $\left(k=1,2, \ldots, C_{\max }\right)$;

Step 3: $Z_{k}$ is amplified by carrier operation and then loaded on particle individuals $P_{i x}, P_{i y}$, and $P_{i z}$ to be searched. The chaotic operator is operated in the original solution space to obtain a new individual position $U_{k}$, wherein $\left(k=1,2, \ldots, C_{\max }\right)$;

$$
U_{k}=X_{x, y, z}+\frac{\left(X_{\max }-X_{\min }\right)}{2} \times\left(2 Z_{k}-1\right) \text {. }
$$

Step 4 : the fitness value $f\left(U_{k}\right)$ of $U_{k}$ is calculated and compared with the fitness value $f(X)$ of $X$ to retain the best solution, and the above process is shown in Algorithm 1:

\section{Weather Forecast Model}

4.1. Meteorological Data. The meteorological data collected in this study are the average mass concentration of $1 \mathrm{~h}$, and the data were converted into 24-hour daily average. The corresponding meteorological data in this area are 18 statistical variables commonly used in meteorology, as given in Table 1 .

Table 1 provides the important indicators monitored in meteorological information which are important for predicting actual meteorological values. Through the index research of input variables, the corresponding values, and units of different indexes, Table 2 provides the important indexes of predicted output values.

4.2. Weight Analysis and Model Evaluation. The input variable prediction fusion method is to fuse 18 input variables, and the fused variables can effectively analyze the effectiveness of the model proposed in this study.

4.2.1. PCA Weight. $w$ represents the coefficient of each principal component, $w_{i j}$ represents the coefficient of the $j^{\text {th }}$ index of the first principal component, $f_{i}$ represents the variance contribution rate of the first principal component, and the weight of the $q^{\text {th }}$ index is

$$
r_{q}=\frac{\sum_{i=1}^{n} w_{i q} \cdot f_{i}}{\sum_{i=1}^{n} f_{i}}
$$

Normalization of equation (19) is

$$
w_{q}=\frac{r_{q}}{\sum_{i=1}^{m} r_{i}} \text {. }
$$

4.2.2. Entropy Weight. The analysis method of the entropy weight method is to judge the weight based on the variation of index. By calculating the information entropy, the weight relationship is analyzed by the information entropy of each index. When the information entropy of the index is relatively small, it means that the variation probability of the index is relatively large, the amount of information that can be reflected is relatively large, and the representative weight in the whole index evaluation system is relatively large. If the information entropy of the index is relatively large, it means that the variation probability of the index is small, the amount of information that can be reflected is small, and the representative weight in the whole index evaluation system is relatively small.

$$
\begin{aligned}
& h_{i}=-\frac{1}{\ln n} \sum_{j=1}^{n} \frac{r_{i j}}{\sum_{j=1}^{n} r_{i j}} \ln f_{i j}, \\
& w_{i}=\frac{1-h_{i}}{m-\sum_{i=1}^{m} h_{i}}, \quad\left(0 \leq 1, \sum_{i=1}^{m} w_{i}=1\right) .
\end{aligned}
$$

4.2.3. Correlation Analysis. Through the analysis of the correlation of input variables, it is mainly to analyze which input indicators have input weight relationships among different output variables. We adopt the correlation formula as follows:

$$
\rho(x, y)=\frac{\operatorname{Cov}(x, y)}{[V(x) \cdot V(y)]^{0.5}} .
$$

According to the correlation coefficients between the calculated meteorological parameters, the appropriate input can be selected when constructing the particle algorithm, which can not only reduce the parameters of model training but also avoid the negative impact of unnecessary parameters on the prediction results.

4.2.4. Model Evaluation. In order to comprehensively evaluate the prediction accuracy of the model, three types of evaluation indexes, namely, root mean square error (RMSE), average absolute error (MAD), and determination coefficient $\left(R^{2}\right)$, are used to evaluate the difference level, absolute error, and goodness-of-fit between the predicted value and the measured value of the model.

$$
\begin{aligned}
\mathrm{RMSE} & =\sqrt{\frac{1}{n} \sum_{i=1}^{n}\left(y_{i}-\widehat{y}_{i}\right)^{2},} \\
\mathrm{MAE} & =\frac{1}{N} \sum_{i=1}^{n}\left|y_{i}-\widehat{y}_{i}\right|, \\
R^{2} & =\frac{\sum_{i=1}^{n}\left(y_{i}-\bar{y}\right)^{2}-\sum_{i=1}^{n}\left(y_{i}-\hat{y}_{i}\right)^{2}}{\sum_{i=1}^{n}\left(y_{i}-\bar{y}\right)^{2}} .
\end{aligned}
$$


Step 1: algorithm parameter setting: PSO scale $N$, maximum iteration times $T_{\max }$, iteration times $t$;

Step 2: for population initialization, let $t=0$;

Step 3: perform iteration on that particle swarm optimization algorithm;

While $\left(t<T_{\max }\right)$ do

Calculate the fitness value of particle swarm, update the positions of $p_{\text {best }}$ and $t_{\text {best }}$ according to the fitness value, and record the positions $P_{i x}, P_{i y}$, and $P_{i z}$;

For $i=1$ to $N$ do

Calculate the speed and position values of control parameters according to equations (1)-(4);

Update the initialization population coded by parameter coordinates according to equations (11)-(13);

Update the positions of the remaining particles according to equations (4)-(6);

Update the position of $P_{i x}, P_{i y}$, and $P_{i z}$ according to Kent chaotic search strategy;

End for

$t=t+1$

End while

Algorithm 1: ADPSO algorithm pseudocode.

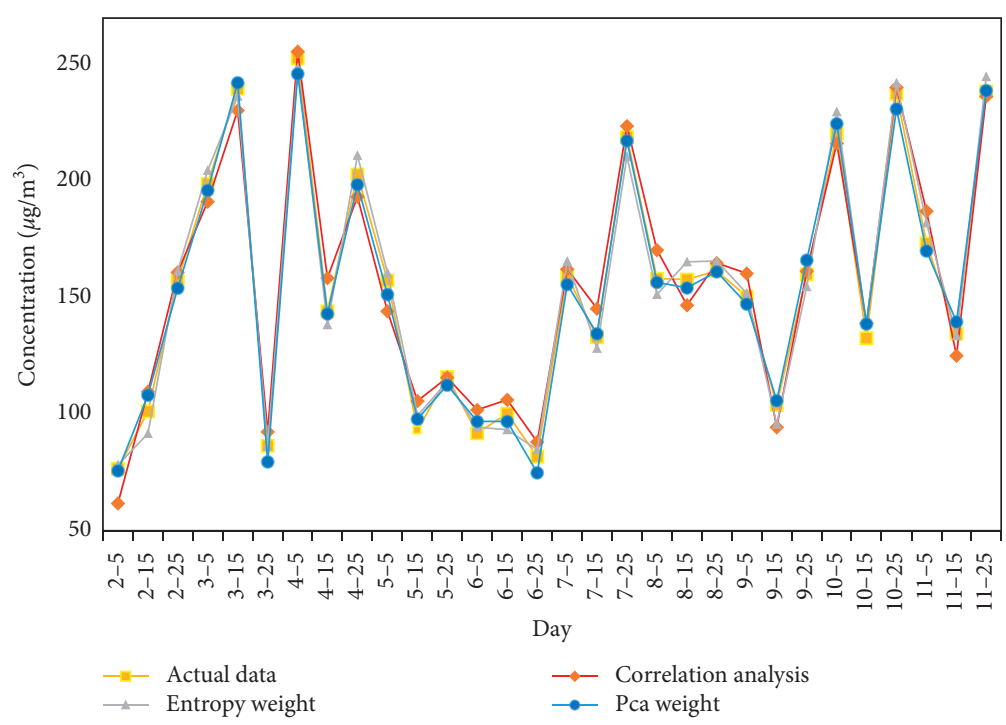

Figure 2: Prediction results of different weights under PSO prediction.

There are differences in the adoption methods of the above three methods. For the judgment and correlation analysis of all input indexes of the first two methods, only the better indexes may be selected for input research to see how the final prediction results are. The PSO algorithm is used to predict PM2.5 in meteorology under different weights, and the prediction effect is shown in Figure 2.

As can be seen from Figure 1, the weight index application using PCA weight has the best performance, is highly consistent with actual data on the whole, and has a good overall prediction effect. However, the worst prediction effect is the related index relationship model, which may lack some input attributes, and the overall performance is not ideal.

In order to reflect the different advantages of the three evaluation methods, the performance of the above three evaluation models under the PSO algorithm is compared by using formulas (23)-(25), as shown in Figure 3.

Figure 3 shows the advantages and disadvantages of evaluation indexes under different weights. It is obvious that PCA weight has the best performance and correlation analysis model has the worst performance. On the whole, the prediction effect is relatively ideal. In the following, various algorithms and the PCA weight model are used to comprehensively predict different index weights.

4.3. Comparison of Prediction Methods of Different Algorithms. Considering that the adaptive optimization particle swarm optimization algorithm proposed in this study has certain advantages in the above three weight relationships, PCA weight relationship is used to set the weights of the other three algorithms proposed in this section. Compare the prediction effects of other algorithms under meteorological data, as shown in Figure 4.

From Figure 4, it can be seen that the ADPSO algorithm has obvious advantages in the overall prediction effect, and its good performance on dates 6-5 and 6-15 basically coincides with the original data. The prediction effect is not ideal for the GA model, and the prediction effect is the worst on dates $3-5,3-25,10-15$, and $10-25$, which is almost close 


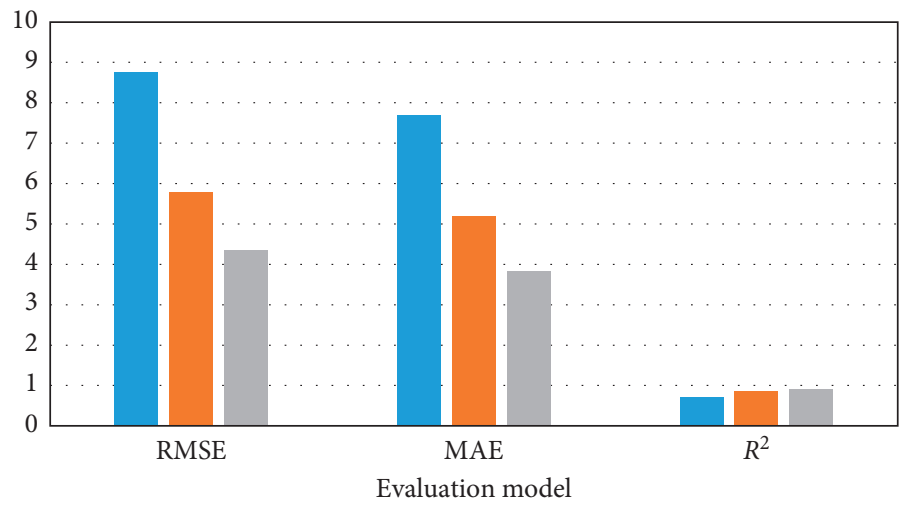

- Correlation analysis

- Entropy weight

- PCA weight

Figure 3: Comparison of evaluation indexes under different weights.

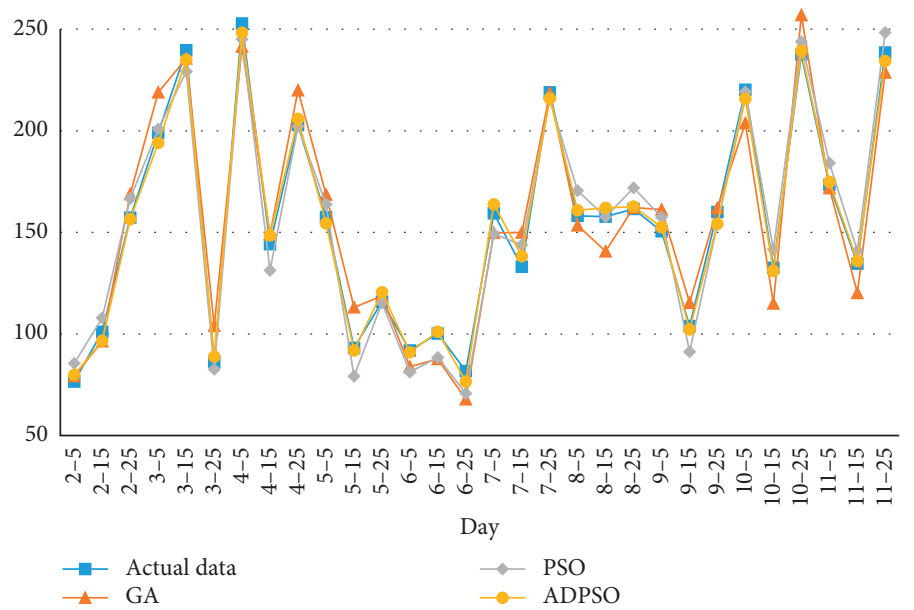

FIgURE 4: Comparison of the ADPSO algorithm with other algorithm prediction.

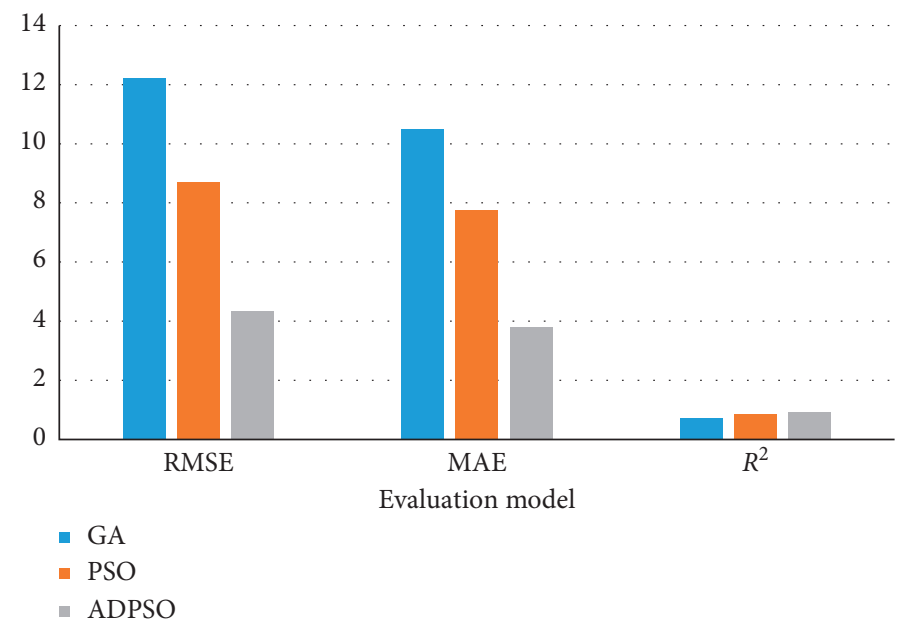

FIGURE 5: Comparison of evaluation models of different algorithms.

to the difference of 20 . The average prediction difference of the GA model is greater than 10, the average prediction difference of the PSO model is 8 , and the average prediction difference of the ADPSO model is 3 . Then, the specific effects of different algorithms are analyzed through model evaluation, as shown in Figure 5. 
As can be seen from Figure 5, the overall performance of ADPSO is relatively advantageous, and the prediction effect is most obvious compared with other algorithms.

\section{Conclusion}

In this study, an improved particle swarm optimization algorithm is proposed to predict meteorological data under the influence of the GNSS, and the proposed method has obvious advantages in the prediction effect and performance. Different evaluation models also have different differences when comparing the same kind of algorithms, which may be related to the variability of data input or different dimensions. When evaluating the model, the better weight relationship is used to predict the model. Considering the later research work, the application prediction of other adaptive algorithms is deeply analyzed, and the execution time and prediction accuracy are improved. Furthermore, the evaluation and prediction of the ADPSO algorithm under other meteorological indicators can improve the prediction accuracy of the algorithm and comprehensively apply the evaluation under different scenes and nonmeteorological factors.

\section{Data Availability}

The data used to support the findings of this study are available from the corresponding author upon request.

\section{Conflicts of Interest}

The authors declare that they have no conflicts of interest.

\section{References}

[1] A. Rovira-Garcia, D. Ibáñez-Segura, R. Orús-Perez, J. M. Juan, J. Sanz, and G. González-Casado, "Assing the quality of ionospheric models through GNSS positioning error: methodology and results," GPS Solutions, vol. 24, no. 1, pp. 1-12, 2020.

[2] Y. B. Yao, Q. Z. Zhao, and B. Zhang, "A method to improve the utilization of GNSS observation for water vapor tomography," Annales Geophysicae, vol. 34, no. 1, pp. 143-152, 2016.

[3] G. L. Stephens and C. D. Kummerow, "The remote sensing of clouds and precipitation from space: a review," Journal of the Atmospheric Sciences, vol. 64, no. 11, pp. 3742-3765, 2007.

[4] J. Duan, M. Bevis, P. Fang et al., "GPS meteorology: direct estimation of the absolute value of precipitable water," Journal of Applied Meteorology, vol. 35, no. 6, pp. 830-838, 1996.

[5] J. Boehm, A. Niell, P. Tregoning, and H. Schuh, "Global mapping function (GMF): a new empirical mapping function based on numerical weather model data," Geophysical Research Letters, vol. 33, no. 7, pp. 5-18, 2006.

[6] J. Haase, M. Ge, and H. Vedel, "Accuracy and variability on GPSTroposthetic delay mesurements of water vapor in the western mediterranean," American Meteorological Society, vol. 42, pp. 1547-1568, 2003.

[7] J. Fidalgo, M. Cueto, E. Sardón, and A. Cezón, "Ionospheric prediction over equatorial and non-equatorial regions using GNSS data," in Proceedings of the 28th International Technical Meeting of the Satellite Division of the Institute of Navigation, pp. 3565-3577, Tampa, Florida, September 2015.
[8] S. de Haan, "Assimilation of GNSS ZTD and radar radial velocity for the benefit of very-short-range regional weather forecasts," Quarterly Journal of the Royal Meteorological Society, vol. 139, no. 677, pp. 2097-2107, 2013.

[9] R. Singh, S. P. Ojha, N. Puviarasan, and V. Singh, "Impact of GNSS signal delay association on short range weather forecasts over the Indian region," Journal of Geophysical Research: Atmospheres, vol. 124, no. 17-18, pp. 9855-9873, 2019.

[10] A. Karabatic, R. Weber, and T. Haiden, "Near real-time estimation of tropospheric water vapour content from ground based GNSS data and its potential contribution to weather now-casting in Austria," Advances in Space Research, vol. 47, no. 10, pp. 1691-1703, 2011.

[11] F. Huang, J. L. Garrison, N. Rodriguez-Alvarez et al., "Sequential processing of GNSS-R delay-doppler maps to estimate the ocean surface wind field," IEEE Transactions on Geoscience and Remote Sensing, vol. 57, no. 12, pp. 1020210217, 2019.

[12] J. Juang, "Assessment of regional satellite navigation systems in GNSS reflection experiences," Proceedings of the Ion Pacific PNT Meeting, vol. 8900, no. 6, pp. 36-40, 2013.

[13] L. Li, Z. Yuan, P. Luo et al., "A system developed for monitoring and analyzing dynamic changes of GNSS precipitable water vapor and its application," in Proceedings of the China Satellite Navigation Conference (CSNC) 2015, pp. 95-105, Xian, China, May 2015.

[14] T. Ning, J. Wang, G. Elgered et al., "The uncertainty of the atmospheric integrated water vapour estimated from GNSS observations," Atmospheric Measurement Techniques, vol. 9, no. 1, pp. 79-92, 2016.

[15] S. W. Lee, J. Kouba, B. Schutz, D. Kim, and Y. J. Lee, "Monitoring predictable water vapor in real-time using global navigation satellite systems," Journal of Geodesy, vol. 87, no. 10-12, pp. 923-934, 2013.

[16] P. Mateus, P. M. A. Miranda, G. Nico, J. Catalão, P. Pinto, and R. Tomé, "Assimilating InSAR maps of water vapor to improve heavy rainfall forecasts: a case study with two successive storms," Journal of Geophysical Research: Atmospheres, vol. 123, no. 7, pp. 3341-3355, 2018.

[17] X. Wang, K. Zhang, S. Wu et al., "The correlation between GNSS-derived predictable water vapor and sea surface temperature and its responses to El Nio-southern oscillation," Remote Sensing of Environment, vol. 216, pp. 1-12, 2018.

[18] N. Jakowski, B. Yannick, G. D. Franceschi et al., "Monitoring, tracking and forecasting ionospherical perturbations using GNSS techniques," Journal of Space Weather and Space Climate, vol. 2, no. A4, Article ID A260000, 2012.

[19] G. Chen and Z. Li, "Improved particle swarm optimization LSSVM spatial location trajectory data prediction model in health care monitoring system," Personal and Ubiquitous Computing, pp. 1-10, 2019. 